Vera Lúcia Lopes Barros 1 José Manuel Macário Rebêlo 2 Francinal do Soares Silva 2

\section{Flebotomíneos (Diptera, Psychodidae) de capoeira do Município do Paço do Lumiar, Estado do Maranhão, Brasil. Área de transmissão de leishmaniose}

\author{
Sandflies (Diptera, Psychodidae) \\ in a secondary forest area in the county \\ of Paço do Lumiar, Maranhão, Brazil: \\ a leishmaniasis transmission area
}

\footnotetext{
1 Departamento de Biologia, Universidade Estadual do Maranhão. Praça Madre Deus 2, São Luís, MA 65025-560, Brasil. 2 Núcleo de Patologia Tropical e Medicina Social, Departamento de Patologia, Universidade Federal do Maranhão. Praça Madre Deus 2 São Luís, MA 65025-560, Brasil.
}

\begin{abstract}
This paper analyzes the weal th of species, relative abundance, seasonal fluctuation, and nocturnal activity of sandflies. The field survey was conducted in a "capoei ra" (secondary forest) area in the county of Paço do Lumiar, Maranhão, where cutaneous and transmission of visceral leishmaniasis frequently occurs. Sandflies were captured by CDC-type light traps from 6:00 PM to 6:00 AM, oncea month, from March 1997 to February 1998. A total of 489 specimens were collected ( 251 males and 238 females), distributed among 10 species: Lutzomyia antunesi (45.19\%), Lutzomyia whitmani (29.4\%), Lutzomyia Iongipalpis (7.56\%), Lutzomyia sordelli (6.34\%), Lutzomyia flaviscutellata (4.5\%), Brumptomyia avellari (4.09\%), Lutzomyia evandroi ( $1.85 \%)$, Lutzomyia umbratilis $(0.61 \%)$, Lutzomyia corossoniensis $(0.41 \%)$, and Lutzomyia trispinosa $(0.41 \%)$. The sandflies were present year round, with higher abundance during the rainy season. They were present in all intervals studied, with the highest frequency between 12:00 PM and 1:00 AM (31\%).
\end{abstract}

Key words Lutzomyia; Phlebotominae; Psychodidae; Leishmaniasis; Insect Vectors

Resumo Apresentam-sea riqueza de espécies, a abundância relativa, a flutuação sazonal e horária dos flebótomos estudados em uma capoeira da Il ha de São Luís - MA, área de transmissão de lei shmani oses tegumentar e visceral. Os flebótomos foram capturados em armadi lha luminosa, das 18h às 6h, uma vez por mês, de março de 1997 a feverei ro de 1998. Foram coletados 489 espécimens (251 machos e 238 fêmeas) de dez espéci es: Lutzomyia antunesi (45,19\%), Lutzomyi whitmani (29,4\%), Lutzomyia longipalpis (7,56\%), Lutzomyia sordellii (6,34\%), Lutzomyia flaviscutellata (4,5\%), Brumptomyia avellari (4,09\%), Lutzomyia evandroi (1,85\%), Lutzomyia umbratilis $(0,61 \%)$, Lutzomyia corossoniensis $(0,41 \%)$ e Lutzomyia trispinosa $(0,41 \%)$. Os flebótomos esti veram presentes o ano intei ro, com maior abundância na estação chuvosa (janeiro a junho), e em todos os horários, principalmente entre Oh e $1 \mathrm{~h}$.

Palavras-chave Lutzomia; Phlebotomiae; Psychodidae; Leishmanioses; Insetos Vetores 
Introdução

As leishmanioses são atualmente um dos maiores problemas de saúde pública nos Município de São Luís, São José de Ribamar, Paço do Lumiar e Raposa, todos situados na Il ha de São Luís, Estado do Maranhão. Além de constituírem-se em uma das principais endemias, desconhecem-se os vetores da doença e as circunstâncias em que vem ocorrendo o processo de transmissão.

No início da década de 80, a leishmaniose visceral americana (LVA) atingiu o Município de São Luís e, posteriormente, os Municípios de São José de Ribamar e do Paço do Lumiar (Nascimento et al., 1994; Silva et al., 1997). Em 1994, essa enfermidade ocorreu em todos os meses do ano, nos três municípios da Ilha, sendo 135 casos em São Luís, 125 em São José de Ribamar e 66 no Paço do Lumiar.

A leishmaniose tegumentar americana (LTA) também vem adquirindo importância na Ilha de São Luís. Em 1996, Ferreira et al. relataram a existência de, pelo menos, 46 casos de LTA, sendo 32 em São Luís, oito em São José de Ribamar e três em Paço do Lumiar (Ferreira et al., 1996). A maior prevalência incidiu justamente em áreas de expansão periurbana, em virtude da implantação de grandes projetos agropecuários e industriais (Consórcio ALUMAR, Companhia Vale do Rio Doce) e das invasões que vêm ocorrendo ultimamente.

Lutzomyia longi palpis é uma espécie bastante comum na Il ha de São Luís, especialmente nas localidades onde o calazar é endêmico (Nascimento et al., 1994). Pode ser encontrada nas áreas rurais, bem como nos bairros periféricos, fazendo com que a moléstia assuma um caráter urbano. Além de L. Iongi pal pis, existem relatos também da presença de Lutzomyia whitmani, Lutzomyia flavi scutellata, Lutzomyia wellcomei e Lutzomyia umbratilis em áreas de ocorrência de LTA (Rebêlo et al., 1999), todas relacionadas com o peridomicílio na zona rural da llha.

Neste trabalho, fez-se um levantamento das espécies de flebotomíneos numa capoeira do Município de Paço do Lumiar, área de transmissão de LVA e LTA, com o intuito de determinar as espécies que ainda mantêm estoques silvestres, dando a conhecer a flutuação sazonal, a freqüência horária, a riqueza e a abundância relativa das espécies. O propósito é ampliar os conhecimentos sobre a bioecologia desses insetos e auxiliar no controle das leishmanioses na Ilha de São Luís.
Material e métodos

Área de estudo

O estudo foi realizado na periferia de uma capoeira de 68 hectares, na localidade das Mercês, no Município do Paço do Lumiar, Estado do Maranhão. Essa área foi selecionada para o estudo em razão da existência de casos de leishmaniose humana detectados desde a década de 80.

O Município de Paço do Lumiar situa-se a 20 30' LS e 440 7' LW, na Ilha de São Luís, Estado do Maranhão, tendo uma altitude média de $15 \mathrm{~m}$. A vegetação caracteriza-se pela presença de capoeiras e manguezais, sendo intenso o desmatamento que vem acontecendo nas últimas duas décadas, levando ao predomínio de áreas abertas com forte intrusão de babaçu, Orbygnia phalerata (IBGE, 1984). O clima é tropical mesotérmico e úmido, com duas estações bem-definidas. A chuvosa, de janeiro a junho, concentra, em média, cerca de $94 \%$ do total anual das chuvas; a estação seca, de junho a dezembro, concentra apenas $6 \%$. O total pluviométrico médio é de $1.900 \mathrm{~mm}$ anuais. As temperaturas são elevadas durante o ano todo (média de $26^{\circ} \mathrm{C}$ ) com variação anual pequena, principalmente nos meses de abril, maio e junho (IBGE, 1984).

\section{Amostragem}

Os flebótomos foram capturados em duas armadilhas luminosas tipo CDC, instaladas em uma clareira da capoeira a 1,5 $\mathrm{m}$ de altura e 6 $m$ de distância entre si, no período de março de 1997 a fevereiro de 1998, uma vez por mês, das $18 \mathrm{~h}$ às $6 \mathrm{~h}-12$ horas ininterruptas -, totalizando 144 horas. Os flebótomos capturados a cada hora eram acondicionados em câmaras mortíferas à base de acetato de etila e em seguida conservados em tubos de ensaio contendo álcool a 70\%. No laboratório, o material foi montado entre lâmina e lamínula e identificado seguindo a classificação proposta por Young \& Duncan (1994).

Utilizou-se o teste do Qui-quadrado para analisar as diferenças na densidade dos flebótomos entre as estações seca e chuvosa, assim como entre os horários nos quais foram encontrados. As diferenças foram consideradas significativas quando a probabilidade ( $p$ ) do erro foi inferior a $1 \%(p<0,01)$. 


\section{Resultados}

Riqueza de espécies e abundância relativa

Foram coletados 489 espécimens ( 251 machos e 238 fêmeas) de flebótomos de dez espécies, a saber: Lutzomyia antunesi Coutinho, 1939, Lutzomyia corossoniensis Le Pont e Pajot, 1978, Lutzomyia evandroi Costa Lima e Antunes, 1936, Lutzomyia flaviscutellata Mangabeira, 1942, Lutzomyia Iongipalpis Lutz e Neiva, 1912, Lutzomyia sordellii Shannon e Del Ponte, 1927, Lutzomyia trispinosa Mangabeira, 1942, Lutzomyia umbratilis Ward e Frahia, 1977, Lutzomyia whitmani Antunes e Coutinho, 1939 e Brumptomyia avellari Costa Lima, 1932. L. antunesi foi a espécie mais freqüente na área de estudo, sendo representada por $45,19 \%$ dos espécimens capturados. Seguem-se, na ordem de prevalência, L. whitmani $(29,04 \%)$, L. longi palpis (7,56\%), L. sordellii (6,34\%), L. flaviscutellata (4,50\%) e B. avellari (4,09\%). As demais espécies representaram juntas 3,28\%. As duas espécies mais abundantes na área, L. antunesi e L. whitmani, representaram 74,23\%, sendo, portanto, mais freqüentes do que todas as outras juntas (Tabela 1).

\section{Flutuação sazonal}

A riqueza de espécies variou durante o ano, sendo menor do final da estação chuvosa (maio) ao início da estação seca (agosto). Nos meses desse período de transição, foi registrada a ocorrência de duas a quatro espécies, repre- sentando menos de $50 \%$ daquelas conhecidas na área. Já de setembro a abril, que inclui a maior parte dos períodos seco e chuvoso, o número de espécies presente em cada mês foi sempre igual ou superior a cinco. Portanto, mais de $50 \%$ das espécies estavam presentes na área na maior parte do ano (Tabela 2).

Com relação à freqüência sazonal dos flebotomíneos, foram observadas diferenças estatisticamente significativas ( $p \varangle 0,01$ ) nos percentuais de espécimens encontrados entre as estações, sendo 60\% (294 espécimens) capturados na estação chuvosa (janeiro/junho) e 40\% (195 espécimens) na estação seca (julho/ dezembro). Apesar de os flebótomos predominarem no período chuvoso, a distribuição dos espécimens foi mais uniforme no período seco.

No período chuvoso, a freqüência de espécimens no mês de março foi significativamente maior ( $p \varangle 0,01$ ) do que nos demais meses. Além disso, o maior número de espécies e de espécimens capturados foi coincidente no mês de março; já no período seco, não se observou tal padrão, pois o maior número de espécies foi encontrado em outubro e o de espécimens em novembro.

L. antunesi e L. whitmani ocorreram o ano inteiro, ambas com mais freqüência no mês de março (Tabela 2). L. longi pal pis apresentou um pico de fevereiro a março, decaindo sensivelmente até desaparecer em novembro/ dezembro. L. sordellii e B. avellari apresentaram padrões similares de ocorrência, estando presentes de meados do período seco até meados do

Tabela 1

Espécies de Phlebotominae encontradas numa área de capoeira da localidade das Mercês, Município do Paço do Lumiar, Maranhão, 1997-1998.

\begin{tabular}{lcccr}
\hline Flebótomos & Machos & Fêmeas & Indivíduos & $\%$ \\
\hline L. antunesi & 169 & 52 & 221 & 45,19 \\
L. corossoniensis & - & 2 & 2 & 0,41 \\
L. evandroi & 1 & 8 & 22 & 1,85 \\
L. flaviscutellata & - & 22 & 37 & 4,50 \\
L. longipalpis & 17 & 20 & 31 & 7,56 \\
L. sordellii & - & 31 & 2 & 6,34 \\
L. trispinosa & 2 & - & 3 & 0,41 \\
L. umbratilis & - & 3 & 142 & 0,61 \\
L. whitmani & 42 & 100 & 20 & 29,04 \\
B. avellari & 20 & - & 489 & 4,09 \\
Espécimens & 251 & 238 & 100,0 & 100,0 \\
\% & 51,3 & 48,7 & & \\
\hline
\end{tabular}


Tabela 2

Freqüência mensal das espécies de Phlebotominae encontradas numa área de capoeira da localidade das Mercês, Município do Paço do Lumiar, Maranhão, 1997-1998.

\begin{tabular}{|c|c|c|c|c|c|c|c|c|c|c|c|c|c|}
\hline & \multicolumn{6}{|c|}{ Estacão chuvosa } & \multicolumn{6}{|c|}{ Estação seca } & \multirow[t]{2}{*}{ Total } \\
\hline & jan & fev & mar & $a b r$ & mai & jun & jul & ago & set & out & nov & dez & \\
\hline L. antunesi & 11 & 17 & 82 & 3 & 3 & 10 & 10 & 22 & 10 & 4 & 42 & 7 & 221 \\
\hline L. corossoniensis & - & - & 2 & - & - & - & - & - & - & - & - & - & 2 \\
\hline L. evandroi & - & - & - & - & - & 1 & - & 3 & - & 1 & 4 & - & 9 \\
\hline L. flaviscutellata & 5 & - & 6 & - & - & 1 & - & - & - & 1 & 7 & 2 & 22 \\
\hline L. longipalpis & - & 21 & 12 & 1 & - & 1 & 1 & - & 1 & - & - & - & 37 \\
\hline L. sordellii & 8 & 8 & 3 & 5 & - & - & - & - & - & 4 & 1 & 2 & 31 \\
\hline L. trispinosa & - & - & - & 1 & - & - & - & - & 1 & - & - & - & 2 \\
\hline L. umbratilis & - & - & 2 & - & - & - & - & - & - & 1 & - & - & 3 \\
\hline L. whitmani & 9 & 3 & 58 & 1 & 14 & - & 20 & 6 & 8 & 5 & 13 & 5 & 142 \\
\hline B. avellari & 5 & 1 & - & - & - & - & - & - & 2 & 1 & 5 & 6 & 20 \\
\hline Espécimens & 38 & 50 & 165 & 11 & 17 & 13 & 31 & 31 & 22 & 17 & 72 & 22 & 489 \\
\hline Espécies & 5 & 5 & 7 & 5 & 2 & 4 & 3 & 3 & 5 & 7 & 6 & 5 & \\
\hline
\end{tabular}

período chuvoso. Não foram detectadas nas armadilhas durante cinco meses ou mais. L. flaviscutellata tendeu a ocorrer irregularmente o ano inteiro, em meses alternados, sendo as maiores freqüências registradas nos meses de janeiro, março e novembro. As espécies L. trispinosa e L. umbrati lis ocorreram apenas dois meses durante todo o ano, sendo um mês no período chuvoso e o outro no período seco. Lutzomyia evandroi ocorreu predominantemente no período seco.

Freqüência horária

Os flebótomos estiveram presentes em todos os horários (Tabela 3). As espécies que apresentaram períodos mais amplos de atividade foram L. antunesi , L. whitmani, L. Iongipalpis, L. sordellii, L. flaviscutellata e B. avel lari, mas somente as duas primeiras ocorreram rigorosamente em todos os horários. As maiores freqüências de flebótomos capturados foram registradas entre 0 h e 1 h, tanto de espécimens, quanto de espécies. O número de espécimens encontrados entre $0 \mathrm{~h}$ e $\mathrm{Ih}$ foi significativamente maior $(p<0,01)$ do que em qualquer outro horário. Já a distribuição das espécies foi mais homogênea, variando de quatro a oito na primeira metade da noite e de cinco a nove na segunda metade.

L. antunesi iniciou sua atividade com moderada freqüência, diminuindo depois das $21 \mathrm{~h}$, para ressurgir com alta densidade entre oh e 1h, declinando em seguida até às $6 \mathrm{~h}$. Padrão similar foi observado para L. whitmani e L. fla- viscutellata. Já L. longi palpis e L. sordellii apresentaram freqüências mais regulares na maioria dos horários. Entretanto, as maiores freqüências foram observadas depois das 22h, com pico às 3h. B. avellari apresentou-se com mais freqüência depois das $2 \mathrm{~h}$ e não foi encontrada nos dois primeiros horários (Tabela 3). L. trispinosa ocorreu nas primeiras horas da primeira metade noite e $L$. umbratilis nas primeiras horas da segunda metade. Já L. evandroi esteve presente nos quatro primeiros horários e irregularmente na segunda metade da noite.

\section{Discussão}

As espécies de flebótomos encontradas na capoeira das Mercês representam aproximadamente $45,5 \%$ das de ocorrência conhecida na Ilha de São Luís. Entre as espécies encontradas neste estudo, somente L. corossoniensis, L. sordellii, L. trispinosa e B. avellari ainda não foram encontradas nos ambientes domiciliares. Esses dados associados àqueles relatados por Rebêlo et al. (1999) possibilitaram uma nova abordagem sobre a distribuição dos flebótomos na llha, pois mostram a existência de uma fauna diversificada, com espécies restritas ao ambiente silvestre e outras aparecendo em habitações rurais e até mesmo em bairros periféricos da zona urbana.

A maioria das espécies foi encontrada irregularmente distribuída durante o ano. Nenhuma comportou-se como espécie sazonal, e somente duas espécies foram consi deradas anu- 
ais, L. antunesi e L. whitmani, as quais estiveram presentes o ano inteiro e também durante toda a noite. A ocorrência desta última espécie o ano inteiro é particularmente importante, em virtude do seu reconhecido papel como vetor de Leishmania. É uma das espécies mais comuns nos ambientes silvestres e domiciliares em áreas rurais da Amazônia maranhense (Ready et al., 1997) e da llha (Rebêlo et al., 1999).

A presença de L. flaviscutellata na capoeira das Mercês revestiu-se de grande significado epidemiológico, pois esse flebótomo pode atuar como vetor da Leishmania. amazonensis, o agente responsável pela maioria dos casos de leishmaniose cutânea difusa (LCD) no Estado do Maranhão (Costa et al., 1992). Essa espécie era consi derada estritamente silvestre (Tal hari et al., 1988), mas, ao que parece, na Ilha de São Luís, ela vem adaptando-se não só às áreas de vegetação secundária (capoeira), como ao ambiente domiciliar (Rebêlo et al., 1999).

L. umbratilis tem sido encontrada no Maranhão freqüentemente associada com áreas silvestres (Rebêlo et al., 1996), embora possa ser encontrada em baixa freqüência no ambiente domiciliar (Rebêlo et al., 1999). É uma espécie epidemiologicamente importante na região amazônica, na qual o Maranhão está incluído, pois transmite Leishmania guyanensis, um dos agentes etiológicos da LTA (Lainson \& Shaw, 1987).

Nos estudos realizados, até o momento, no Maranhão, os flebótomos L. corossoni ensis, L. sordellii, B. avellari e L. trispinosa só foram encontrados no ambiente silvestre, sendo este o primeiro registro na Ilha de São Luís para as três primeiras espécies. L. longipal pis tem sido a mais freqüente das espécies encontradas no ambiente domiciliar e, talvez, a mais bem adaptada ao convívio com o homem e os animais domésticos em áreas de endemismo de LVA (Nascimento et al., 1994; Rebêlo et al., 1999). Ela se encontra distribuída em quase toda a Ilha, incluindo as zonas periféricas dos aglomerados urbanos, as proximidades da orla litorânea, no nível do mar elocais descampados com franca ventilação, fugindo, desse modo, das características epidemiológicas apresentadas nas demais localidades da região neotropical.

O padrão de ocorrência dos flebótomos, ao longo do ano, na capoeira das Mercês parece indicar que tanto o período chuvoso, quanto o seco, favorecem o desenvolvimento da maioria das espécies. Isso porque a Ilha de São Luís está situada na zona tropical equatorial, de baixa altitude e com os valores térmicos e de umidade pouco variáveis durante o ano. O fator que tem influência mais direta na flutuação anual dos flebótomos seria a precipitação pluviométrica, já que o pico no número de indivíduos da maioria das espécies foi observado no período chuvoso.

Levando-se em conta os horários de maior atividade das espécies, pode-se verificar que o intervalo compreendido entre $0 \mathrm{~h}$ e $\mathrm{lh}$ representaria o período de preferência para várias

\section{Tabela 3}

Freqüência das espécies de Phlebotominae encontradas numa área de capoeira da localidade das Mercês, Município do Paço do Lumiar, Maranhão, 1997-1998.

\begin{tabular}{|c|c|c|c|c|c|c|c|c|c|c|c|c|c|}
\hline \multirow[t]{3}{*}{ Flebótomos } & \multicolumn{12}{|c|}{ Horários } & \multirow[t]{3}{*}{ Total } \\
\hline & 18 & 19 & 20 & 21 & 22 & 23 & 00 & 01 & 02 & 03 & 04 & 05 & \\
\hline & 19 & 20 & 21 & 22 & 23 & 00 & 01 & 02 & 03 & 04 & 05 & 06 & \\
\hline L. antunesi & 18 & 20 & 19 & 11 & 9 & 11 & 63 & 22 & 29 & 9 & 5 & 5 & 221 \\
\hline L. corossoniensis & - & - & - & 1 & - & - & 1 & - & - & - & - & - & 2 \\
\hline L. evandroi & 2 & 1 & 1 & 1 & - & - & 2 & - & - & 1 & 1 & - & 9 \\
\hline L. flaviscutellata & 1 & 3 & 1 & 2 & 2 & - & 8 & 2 & - & 2 & 1 & - & 22 \\
\hline L. longipalpis & 3 & 2 & 1 & 3 & - & 6 & 4 & 4 & 1 & 7 & 2 & 4 & 37 \\
\hline L. sordellii & 2 & - & 2 & 1 & 5 & 4 & 1 & - & 4 & 6 & 3 & 3 & 31 \\
\hline L. trispinosa & 1 & - & 1 & - & - & - & - & - & - & - & - & - & 2 \\
\hline L. umbratilis & - & - & - & - & - & - & 2 & 1 & - & - & - & - & 3 \\
\hline L. whitmani & 8 & 23 & 8 & 6 & 8 & 7 & 48 & 6 & 13 & 9 & 3 & 3 & 142 \\
\hline B. avellari & - & - & 2 & 1 & 1 & - & 1 & 1 & 7 & 3 & 3 & 1 & 20 \\
\hline Espécimens & 35 & 49 & 35 & 26 & 25 & 28 & 130 & 36 & 54 & 37 & 18 & 16 & 489 \\
\hline Espécies & 7 & 5 & 8 & 8 & 5 & 4 & 9 & 6 & 5 & 7 & 7 & 5 & \\
\hline
\end{tabular}


espécies e, também, o de transmissão da leishmaniose para a população local. Contudo, nesse horário, as pessoas normalmente não têm o hábito de entrar nas capoeiras, a não ser em casos excepcionais, como o dos caçadores.

Segundo depoimento de moradores do sítio das Mercês, a capoeira al i existente, apesar de sofrer constantes modificações antrópicas, ainda serve de hábitat para animais silvestres, como a raposa, mucura (Didel phis) e roedores, que, em vegetação de capoeira, são os mamíferos que mais se destacam. A presença desses animais explica em parte a riqueza e abundância dos flebótomos naquela área.

Concluindo, chama-se a atenção para o fato de que este estudo, planejado para determinar os flebótomos associados com ambientes silvestres, permitiu encontrar algumas espécies que ainda não haviam si do registradas na I lha

\section{Agradecimentos}

À Fundação de Amparo à Pesquisa no Maranhão (FAPEMA), pela concessão da bolsa de iniciação científica para o primeiro autor, e à família Doyhara, por ter cedido gentilmente o seu sítio para a execução deste trabalho.

Referências

COSTA, J. M. L.; SALDANHA, A. C. R.; MELLO-SILVA, A. C.; SERRA NETO, A.; GALVÃO, C. E. S.; PEDROSOSILVA, C. M. \& SILVA. A. R., 1992. Estado atual da leishmaniose cutânea difusa (LCD) no Estado do Maranhão. II. Aspectos epidemiológicos, clínicos e evolutivos. Revista da Sociedade Brasileira de Medicina Tropical, 25:115-123.

FERREIRA, T. C. A.; RODRIGUES Jr., G. F. F.; PINHEIRO, C. R.; SILVA, L. S.; MARQUES, A. L. B.; BRANCO, F. C. \& COSTA, J. M. L., 1996. A leishmaniose tegumentar americana na Ilha de São Luís - Maranhão. In: XIV Jornada de Parasitologia e Medicina Tropical do Maranhão, Resumos, p. 53, São Luís: Universidade Federal do Maranhão.

IBGE (Fundação Instituto Brasileiro de Geografia e Estatística), 1984. Atlas do Maranhão. Rio de Janeiro: Superintendência de Estudos Geográficos e Sócio-Econômicos.

LAINSON, R. \& SHAW, J. J., 1987. Evolution, classification and geographical distribution. In: The Leishmaniasis in Biology and Medicine (W. Peters \& R. Killick-Kendrick, eds.), pp. 1-120, London: Academic Press.

NASCIMENTO, M. D. S. B.; FIORI, B. I. P.; CARNEIRO, L. S.; GONÇALVES FILHO, M. \& BURATINI, M. N., 1994. Estado atual da leishmaniose visceral no Maranhão: Aspectos epidemiológicos preliminares. Revista da Sociedade Brasilei ra de Medicina Tropical, 27 (Sup. 1):242. de São Luís, uma vez que os trabalhos que vêm sendo desenvolvidos restringem-se ao ambiente domiciliar, incluindo-se os de rotina executados pela Fundação Nacional de Saúde (FNSMA). Também permitiu verificar-se que espécies sabidamente comuns no ambiente domiciliar conservam densos estoques silvestres, mostrando o quanto elas estão adaptadas aos diversos ambientes da Ilha. A densidade relativamente alta com que algumas delas ocorreram na capoeira das Mercês é de grande interesse para a vigilância epidemiológica, em razão do risco de reintrodução da infecção leishmaniótica em áreas peri e intradomiciliares submetidas às medidas de controle do calazar e LTA. Os dados levantados devem ser considerados para o aprimoramento das questões de vigilância epidemiológica da endemia na localidade das Mercês, Município do Paço do Lumiar.
REBÊLO, J. M. M.; MENDES, W. A.; COSTA, J. M. L. \& CAVALEIRO, N., 1996. Lista preliminar das espécies do gênero Lutzomyia França 1924 (Psychodidae, Phlebotominae) do Estado do Maranhão, Brasil. Cadernos de Saúde Pública, 12:545-549.

REBÊLO, J. M.; ARAÚJO, J.; CARVALHO, M.; OLIVEIRA, S. T. \& SILVA, F. S., 1999. Flebótomos (Lutzomyia, Phlebotominae) da Ilha de São Luís, zona do Golfão maranhense, Brasil. Revista da Sociedade Brasileira de Medicina Tropical, 32:247-253.

READY, P. D.; DAY, J. C.; SOUZA, A. A.; RANGEL, E. F. \& DAVIES, C. R., 1997. Mitochondrial DNA characterization of populations of Lutzomyia whitmani (Diptera: Psychodidae) incriminated in the peridomestic and silvatic transmission of Leishmania species in Brazil. Bulletin of Entomological Research, 87:187-195.

SILVA, A. R.; VIANA, G. M. C.; VARONIL, C.; PIRES, B. NASCIMENTO, M. D. S. D. \& COSTA, J. M. L., 1997. Leishmaniose visceral (calazar) na llha de São Luís, Maranhão, Brasil: Evolução e perspectivas. Revista da Sociedade Brasileira de Medicina Tropical, 30:359-368.

TALHARI, S.; ARIAS, J. R.; CUNHA, M. G. S.; NAIFF, R. D.; NAIFF, M. F.; FREITAS, R. A. \& BARRETT, T. 1988. Leishmaniose no Estado do Amazonas: Aspectos epidemiológicos, clínicos e terapêuticos. Anais Brasileiros de Dermatologia, 63:433-438. 